\title{
1/ Palliative Therapie eines arteriell blutenden malignen Uretertumors - Transfemorale Tumorembolisation
}

Uretertumoren sind ein insgesamt seltenes Erkrankungsbild (Hohenfellner R et al., Urologie in Klinik und Praxis 1982; 512). Epidemiologisch können bezüglich Inzidenz und Mortalität erhebliche regionale Differenzen auftreten, welche durch unterschiedliche Lebensgewohnheiten (Ernährung, Rauchen, Kaffee) sowie durch die Exposition gegenüber industriellen Noxen (aromatische Amine) erklärt sind. 
In der Mehrzahl der Befunde handelt es sich dabei mit über $90 \%$ um maligne Tumoren. Diese machen insgesamt lediglich 0,8-1\% aller Malignome des Urogenitalsystems aus. Benigne Uretertumoren wie das Papillom sind mit ca. $1 \%$ der Fälle die Ausnahme. Unter den malignen Uretertumoren steht hinsichtlich des histologischen Gewebetyps das Urothelkarzinom mit ca. $90 \%$ an erster Stelle.

In ca. 59\% der Fälle sind die Tumoren im distalen, zu 30\% im mittleren und zu 12\% im proximalen Ureter lokalisiert. Die Geschlechtsverteilung zeigt eine Präferenz der Männer, welche zweimal häufiger als Frauen erkranken. Der Altersgipfel befindet sich in der 7. Lebensdekade (Kühn R et al, Urologe 1994; 33: 247$)$. In $0,5-2 \%$ finden sich bei der Erstdiagnose multiple Tumoren. 2-4\% der Patienten entwickeln zu einem späteren Zeitpunkt einen Tumor im kontralateralen harnableitenden System (Schulze-Seemann W, Lehrbuch Urologie 1995; 213).

Das therapeutische Vorgehen bei supravesikalen Urotheliomen besteht mehrheitlich in der radikalen Nephroureterektomie unter Mitnahme einer Blasenmanschette. Partielle Harnleitersegment-Resektionen sind meist die Ausnahme (Kühn R, et al, Urologe 1994; 33: 247). Einzelne kleine oberflächliche Ureter- bzw. Nierenbeckentumoren können auch ureterorenoskopisch organerhaltend reseziert oder einer Lasertherapie zugeführt werden. Da das Urothelkarzinom der oberen Harnwege ein Erkrankungsbild des höheren Lebensalters mit Erkrankungsgipfel im 7. Dezennium ist, kann die operative Therapie bei den in diesem Lebensalter oftmals multimorbiden Patienten nicht immer zur Anwendung gelangen.

In dem nun Folgenden berichten wir über einen palliativen Therapieansatz durch selektive Tumorembolisation bei einem blutenden Urothelkarzinom des proximalen bis mittleren Ureters.

\section{Fallbericht}

Ein 83-jähriger, männlicher Patient wurde mit einer klinisch manifesten Makrohämaturie stationär aufgenommen. Anamnestisch bekannt war ein Zustand nach transurethraler Resektion eines Blasenkarzinoms (Erstdiagnose: 6/95, pTa, G2) und ein schlecht differenziertes invasives ureterales Karzinom (G3) vom multizentrischen Typ im Bereich des linksseitigen proximalen und mittleren Ureters von ca. $7 \mathrm{~cm}$ Länge (Erstdiagnose: 7/96). Wegen eines Harnaufstaus durch Ureterokklusion zum Zeitpunkt der Erstdiagnose erfolgte nach einer Ureterdilatation die Einlage einer Doppel-J-Ureterschiene mit meist vierteljährlichem Schienen-Wechsel-Intervall, wodurch dem Patienten bisher eine längerfristige Beseitigung des Harnaufstaus ermöglicht wurde. Gleichfalls bestanden eine Niereninsuffizienz im Stadium der kompensierten Retention und eine arterielle Hypertonie.

Über das urogenitale Erkrankungsbild hinaus litt der Patient schon langjährig unter einer therapeutisch sehr schlecht zu behandelnden Kardiomyopathie mit konsekutiver Herzinsuffizienz im Stadium III (NYHA). Ferner bestanden ein Diabetes mellitus Typ IIb und eine Hyperlipidämie.

Aufgrund dieser Multimorbidität und dem damit verbundenen sehr hohen Narkoserisiko (ASA IV) kam eine operative Sanierung des histologisch gesicherten Uretertumors nicht infrage.

Wegen persistierender und hämodynamisch wirksamer Makrohämaturie wurde uns der Patient zu einer angiographisch gesteuerten Tumorembolisation vorgestellt.

Nach Sondierung der linksseitigen Arteria renalis konnte ein kräftiges Tumorgefäß, welches von der unteren Segmentarterie der linken Nierenarterie gespeist wurde, identifiziert werden. Dabei zeigte der aus der unteren Polarterie der linken Nierenarterie speisende arterielle Tumorast eine deutliche Hypertrophie des Gefäßes mit Tumordarstellung (Abb.1). Unter selektiver Sondierung des dilatierten Tumorgefäßes mittels einem gleitbeschichteten 0,018-in Führungsdrahtes gelang die Einlage eines 3-F großen Embolisationskatheters (Leggiero Terumo, Inc., Frankfurt; Abb. 2).

Über diesen wurden die peripheren Tumorgefäße zunächst mit 250$350 \mu \mathrm{m}$ großen Polvinyl-Alkohol-Partikeln embolisiert. Anschließend wurde das Gefäß durch eine zusätzliche Gabe von 0,5 ml einer Suspension eines Gewebeklebers mit öligem Kontrastmittel (Histo-Acryl-Lipiodol) im Mischverhält-

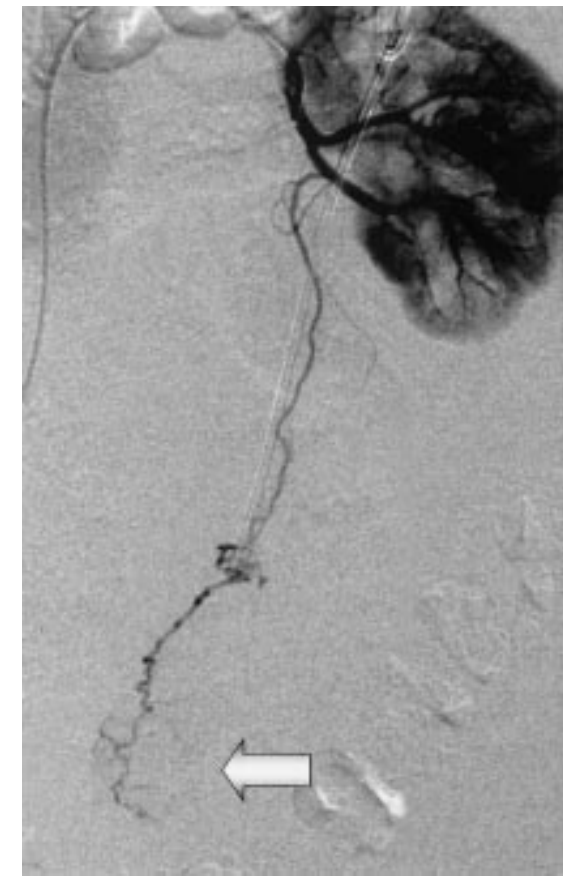

Abb. 1 5-F-Führungskatheter in der linksseitigen Arteria renalis mit Darstellung eines aus der unteren Segmentarterie gespeisten dilatierten Tumorgefäßes mit Tumorkontrastierung (Pfeil); einliegende Doppel-J-Schiene des linken Ureters.

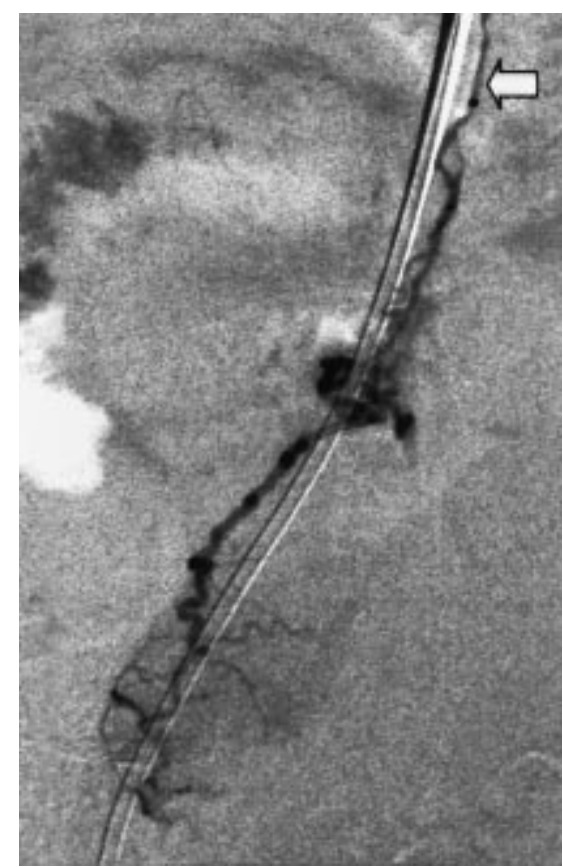

Abb. 2 3-F-Selektivkatheter (Pfeil) im dilatierten Tumorgefäß.

nis $1: 1$ verklebt. Nach unkomplizierter Entfernung des Mikrokatheters wurde die komplette Okklusion des Gefäßes dokumentiert (Abb. 3). 


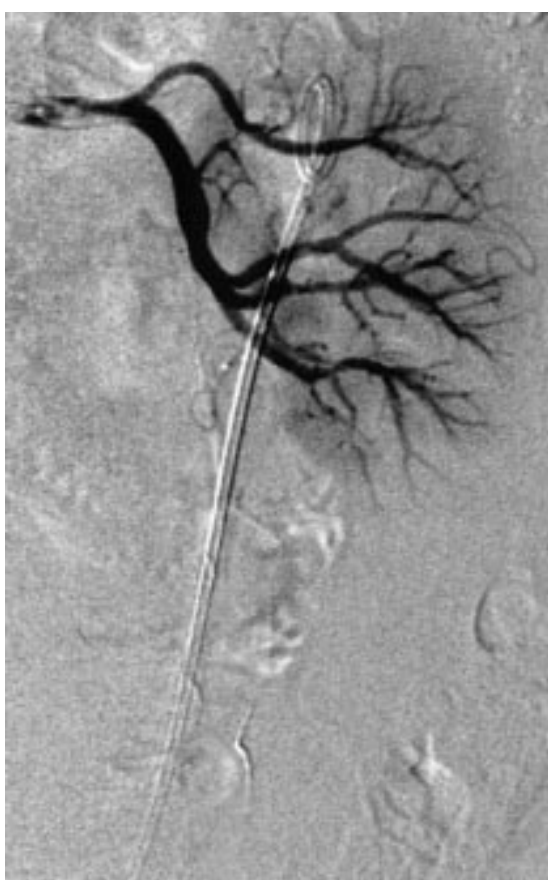

Abb. 3 Dokumentation der vollständigen Gefäß-Okklusion des ehemals von der unteren Polarterie versorgten Tumorastes nach Embolisation rnit Partikeln und Gewebekleber.

18 Stunden später wurde der Druckverband entfernt und der Patient konnte nach Kontrolle seiner Laborparameter beschwerdefrei entlassen werden. Die Kontrolluntersuchungen mit Schienenwechsel - zuletzt ca. 6 Monate nach Embolisation - vergingen ohne Anhalt für erneute Blutungen. Der Patient empfand subjektives Wohlbefinden.

\section{Diskussion}

Urotheliale Tumoren stellen ein seltenes Krankheitsbild dar und sind meist maligne Urothelkarzinome (Hohenfellner R, Zingg EJ. Urologie in Klinik und Praxis; Band I, Diagnostik, Entzündungen und Tumoren. Thieme, Stuttgart · New York 1982; 512 ff). Sie betreffen zumeist das untere oder mittlere Ureterdrittel. Klinisch apparent werden sie nicht selten durch eine Ureterkompression mit Harnaufstau. Durch infiltratives Wachstum mit tumoröser Neovaskularisation kann es im Verlauf zu Tumorblutungen über den Ureter kommen.

Die Therapie der Wahl sollte in der Regel die operative Therapie sein (Kühn R, et al, Urologe 1994; 33: 247). Weil jedoch Uretertumoren erst in fortgeschrittenen Tumorstadien klinisch apparent werden und deshalb spät diagnostiziert werden, können oftmals in Abhängigkeit vom Differenzierungsgrad Atypien Carzinoma in situ und manifeste Karzinome entfernt vom Primärtumor vorkommen [5].

Aus diesen Gründen ist ein ausgedehntes chirurgisches Vorgehen mit Entfernung von ipsilateraler Niere vollständigem Ureter und Ostium unter Mitnahme einer ca. $1 \mathrm{~cm}$ breiten Blasenmanschette Standard.

In der Mehrzahl der Fälle besitzen die Patienten zum Diagnosezeitpunkt ein sehr fortgeschrittenes Lebensalter und einen hierfür oftmals multimorbiden Allgemeinzustand. Unter Berücksichtigung des hierdurch erhöhten Narkoserisikos kann eine operative Therapie nicht in allen Fällen zur Anwendung kommen.

Dementsprechend kam im vorliegenden Fall ein operatives Vorgehen nicht in Betracht, so dass die Indikation zur Tumorembolisation bei einer klinisch apparenten transureteralen Blutung durch ein arrodiertes Tumorgefäß bei bekanntem Uretertumor gestellt wurde.

Die palliative Therapie der Uretertumoren ist mehrfach in der Literatur beschrieben. Zumeist handelt es sich hierbei um eine Beseitigung des tumorinduzierten okkludierenden oder stenosierenden Ureter- bzw. Harn-Aufstaus. In den meisten Fällen erfolgt dazu die Einlage einer Ureterschiene, z. B. eine Doppel-J-Schiene.

Alternativ kann auch bei entsprechend kurzstreckigem Verschluss eine intraureterale Stent-Einlage erfolgen (van Sonnenberg E, et al, Radiologie 1994; 191: 765). Oftmals ist infolge der schnellen Tumorprogression und der dadurch bedingten Stent-Überwachsung oder durch urotheliale hyperplastische Überwucherung die Beseitigung des Harnaufstaus lediglich von kurzer Dauer, so dass bei besserer Praktikabilität die Ureterschiene mit regelmäßigen Wechsel-Intervallen gegenüber dem UreterStent bevorzugt wird (van Sonnenberg E, et al, Radiologie 1994; 191: 765).

Bei fortgeschrittenen Uretertumoren kann es zur Ausbildung von ureterorektalen- oder ureterovaginalen Fisteln mit erheblicher Einschränkung der Lebensqualität kommen.
Als palliatives Therapie-Verfahren ist hier als „ultima ratio“ die transrenale ureterale Okklusion mittels eines hier abgeworfenen Ballons beschrieben (Günther RW, et al, Urol Radiol 1984; 6: 210).

Die arterielle Gefäßversorgung des Ureters erfolgt durch kleine Äste der jeweils in der Nähe liegenden Arterien, welche individuell sehr verschieden ausgebildet sein können. Diese Äste stammen für den Bereich der Pars abdominalis, aus der A. renalis, der Aorta abdominalis der A. testicularis/ductus deferentis (bzw. ovarica/uterina) und der A. iliaca communis. Die Versorgung der Pars pelvina speist sich aus der A. iliaca interna, der A. vesicalis inferior und bei der Frau häufig noch aus der A. uterina (Fleischhauer K [Hrsg.]. Benninghoff - Anatomie Band 2. Urban \& Schwarzenberg 1985; $454 \mathrm{ff})$. Gleichfalls neigen die so versorgenden Gefäße zur Ausbildung von Anastomosen.

Der arterielle Gefäßzugang zu den transureteral blutenden Tumorgefäßen ist somit nur selten möglich und ein technisch anspruchsvolles Verfahren unter Einsatz von Mikrokathetern.

In dem vorliegenden Fall konnte durch ein für den Patienten wenig invasives Verfahren eine komplikationslose und effiziente, schnelle Beseitigung der Tumorblutung erreicht werden, da ein kräftiges Tumorgefäß identifiziert werden konnte.

Als mögliche Komplikation ist neben den für Katheteruntersuchungen üblichen Komplikationen (Ausbildung eines Aneurysma falsum, Nachblutung, Hämatom, etc.) auch mit einer lokalen ureteralen Nekrose durch Unterbindung auch der organernährenden Gefäßabschnitte zu rechnen.

In dem von uns beschriebenen Fall jedoch kam es zu keiner akuten oder chronischen Komplikation mit gutem Palliationsergebnis.

F. Poretti, J. A. Schneider, D. Vorwerk, Ingolstadt 